\title{
Mutagenesis and crystallographic studies of Zymomonas mobilis tRNA-guanine transglycosylase to elucidate the role of serine 103 for enzymatic activity
}

\author{
Ulrich Grädler ${ }^{\mathrm{a}}$, Ralf Ficner ${ }^{\mathrm{b}}$, George A. Garcia ${ }^{\mathrm{c}}$, Milton T. Stubbs ${ }^{\mathrm{a}}$, Gerhard Klebe , $^{\mathrm{a}}$ \\ Klaus Reuter ${ }^{\mathrm{b}, *}$ \\ anstitut für Pharmazeutische Chemie, Philipps-Universität Marburg, Marbacher Weg 6, 35032 Marburg, Germany \\ ${ }^{\mathrm{b}}$ Institut für Molekularbiologie und Tumorforschung, Philipps-Universität Marburg, Emil-Mannkopff-Str. 2, 35037 Marburg, Germany \\ ${ }^{\mathrm{c}}$ Interdepartmental Program in Medicinal Chemistry, College of Pharmacy, University of Michigan, Ann Arbor, MI 48109-1065, USA
}

Received 29 April 1999

\begin{abstract}
The tRNA modifying enzyme tRNA-guanine transglycosylase (TGT) is involved in the exchange of guanine in the first position of the anticodon with $\operatorname{pre}_{1}$ as part of the biosynthesis of the hypermodified base queuine $(Q)$. Mutation of Ser $^{90}$ to an alanine in Escherichia coli TGT leads to a dramatic reduction of enzymatic activity (Reuter, K. et al. (1994) Biochemistry 33, 7041-7046). To further clarify the role of this residue in the catalytic center, we have mutated the corresponding $\mathrm{Ser}^{103}$ of the crystallizable Zymomonas mobilis TGT into alanine. The crystal structure of a TGT(S103A)/preQ $Q_{1}$ complex combined with biochemical data presented in this paper suggest that $\mathrm{Ser}^{103}$ is essential for substrate orientation in the TGT reaction.
\end{abstract}

(C) 1999 Federation of European Biochemical Societies.

Key words: Queuine; Modified nucleoside; tRNA; Crystal structure; Catalytic mechanism

\section{Introduction}

Eubacterial tRNA-guanine transglycosylase (TGT, EC 2.4.2.29) [1] catalyzes the first step of the posttranscriptional modification in the anticodon loop of cognate tRNAs (Asn, Asp, His, Tyr), resulting in the exchange of guanine-34 at the wobble position by the queuine precursor 7-aminomethyl-7deazaguanine $\left(\mathrm{preQ}_{1}\right)$. This precursor is converted to the hypermodified base queuine (Q-base: 7-(4,5-cis-dihydroxy-1-cyclo-penten-3-ylamino-methyl)-7-deazaguanine) in subsequent enzymatic steps [2]. Queuine is found at the wobble position of tRNAs in most organisms with the exception of yeast and archaebacteria [3-5]. In eukaryotes, the Q-base is a nutrient and the replacement of guanine-34 with queuine is carried out directly in one single enzymatic step [6]. The exact biological function of queuine in tRNA is not yet fully understood, but it seems to play a role in the fine tuning of protein biosynthesis in eubacteria [7] and in different cellular events such as development, differentiation, aging and cancer in eukaryotes $[8,9]$. In Shigellae, which are the causative agents of dysentery and effect some 500000 infant deaths per year, the enzymatic activity of TGT is a prerequisite for pathogenicity [10]. This prompted us to launch a detailed biochemical and structural study of this enzyme, since it might be a useful target for the design of a drug against Shigellosis.

*Corresponding author. Fax: (+49) (6421) 28-7008.

E-mail: reuter@imt.uni-marburg.de
The structure of Zymomonas mobilis TGT has been solved at $1.85 \AA$ resolution and revealed a non-canonical $(\beta / \alpha)_{8}$-barrel fold with a zinc binding subdomain. Soaking of TGT crystals with preQ $_{1}$ and inspection of the structure at $2.2 \AA$ resolution indicated a specific $\mathrm{preQ}_{1}$ binding pocket at the C-terminal face of the barrel [11]. Mutagenesis and crystallographic studies revealed Asp ${ }^{102}$ to be the active site nucleophile of $Z$. mobilis TGT in the base exchange reaction. The proposed mechanism involves two consecutive $S_{N} 2$ reactions and the formation of a covalent TGT/tRNA intermediate [12]. An Escherichia coli mutant has been described that contains tRNAs lacking queuine due to a single point mutation in the tgt gene changing the $\operatorname{Ser}^{90}$ codon (E. coli numbering) to a Phe codon [13]. Mutation of $\operatorname{Ser}^{90}$ to cysteine leads to a reduced activity of $E$. coli TGT, mainly due to an increased $K_{\mathrm{M}}$ for the substrate tRNA. The mutation of $\operatorname{Ser}^{90}$ to alanine leads to a dramatic decrease of catalytic efficiency, such that $V_{\max } / K_{\mathrm{M}}$ is reduced by four orders of magnitude compared to the wild-type [14]. The E. coli and Z. mobilis TGT show 53\% sequence identity and have similar kinetic parameters. Inspection of the crystal structure of the $Z$. mobilis TGT in complex with preQ $_{1}$ suggested that $\mathrm{Ser}^{103}$ (which corresponds to $\mathrm{Ser}^{90}$ in E. coli) is involved in substrate orientation. In the preQ $\mathrm{Q}_{1}$ complex, this residue forms a weak hydrogen bond (3.5 $\mathrm{A}$ ) to the 2-NH 2 group of $\mathrm{preQ}_{1}$. Assuming that G34 of the tRNA occupies the $\mathrm{preQ}_{1}$ site during the first step of catalysis, Ser ${ }^{103}$ cannot act as the catalytic nucleophile due to the long distance of $7 \AA$ to nitrogen N9 in preQ $Q_{1}[11]$ and by implication to the 1 ' carbon of the G34 ribose. Although it does not appear to be directly involved in catalysis, the importance of this residue is emphasized by the fact that it seems to be invariant among all suggested and characterized TGT enzymes. This fact is true even for TGT enzymes from archaebacteria and eukaryotes, which exhibit deviating substrate specificities from eubacterial TGTs [15].

To further elucidate the function of this residue in the catalytic center of TGT, we have mutated $\operatorname{Ser}^{103}$ of $Z$. mobilis TGT to alanine in order to determine the crystal structure of the mutated protein. Here we report the biochemical and structural characterization of the $Z$. mobilis TGT(S103A).

\section{Materials and methods}

Reagents and buffers were purchased from Sigma, Aldrich and Gibco-BRL unless stated otherwise. $\left[8-{ }^{3} \mathrm{H}\right]$ Guanine $(50 \mathrm{mCi} / \mathrm{mmol})$ was from Sigma. Oligonucleotides were synthesized at MWG-Biotech. 


\subsection{TGT and E. coli $t R N A^{T y r}$ preparation}

$Z$ mobilis TGT, both wild-type and S103A mutant, were prepared as described previously [16,20] and stored at $4^{\circ} \mathrm{C}$ as microcrystalline suspension. Prior to use, a small sample of the microcrystals was redissolved in a high-salt buffer composed of $10 \mathrm{mM}$ HEPES $(\mathrm{pH}$ 7.5), $10 \mathrm{mM} \mathrm{MgCl}_{2}$ and an appropriate concentration of $\mathrm{NaCl}$. The $\mathrm{NaCl}$ concentration was adjusted to the lowest possible value to obtain a total dissolution of the microcrystals (e.g. $400 \mathrm{mM} \mathrm{NaCl}$ lead to a TGT concentration of $1 \mathrm{mg} / \mathrm{ml}, 2 \mathrm{M} \mathrm{NaCl}$ lead to a maximum TGT concentration of $12 \mathrm{mg} / \mathrm{ml})$. E. coli tRNA ${ }^{\mathrm{Tyr}}-(\mathrm{G} 34)$ was prepared as described previously [14] and stored at a concentration of $10 \mathrm{mg} / \mathrm{ml}$ in a buffer composed of $5 \mathrm{mM}$ cacodylate ( $\mathrm{pH} 6.5$ ), $2 \mathrm{mM} \mathrm{MgCl}_{2}$ and $0.2 \mathrm{mM}$ EDTA.

\subsection{Construction of the TGT mutant S103A}

The construction of the mutant was performed via a polymerase chain reaction (PCR) based on a protocol already described $[17,18]$. The PCR-primers were partially overlapping with both of them containing the mutated codon (Table 1). Successful mutagenesis was verified by sequence analysis of the complete mutated tgt gene using an ABI-PRISM 310 automated sequencer, allowing the identification of clones with no additional undesired mutations.

\subsection{Activity assays}

The determinations of wild-type TGT and TGT(S103A) activities were performed as described previously [16].

\subsection{Band-shift assay}

Wild-type or mutated TGT $(3 \mu \mathrm{M})$ was first incubated with a 20-fold excess of $E$. coli $\mathrm{tRNA}{ }^{\mathrm{Tyr}}$ at $37^{\circ} \mathrm{C}$ for $1 \mathrm{~h}$ in a reaction mixture containing $10 \mathrm{mM}$ HEPES ( $\mathrm{pH} 7.4$ ) and $20 \mathrm{mM} \mathrm{MgCl}_{2}$. The reaction mixtures were then analyzed by native polyacrylamide gel electrophoresis (PAGE) as described previously [12]. Gels were stained with Coomassie brilliant blue R-250 (Merck) followed by silver staining (Sigma).

\subsection{Crystallization and pre $Q_{1}$ soaking}

PreQ $_{1}$ was synthesized as described previously [19]. Wild-type TGT and TGT(S103A) were crystallized as described previously [20]. Crystals were soaked for 3 days at $22^{\circ} \mathrm{C}$ in a buffer composed of $5 \%(\mathrm{w} / \mathrm{v})$ PEG 8000, 10\% (v/v) DMSO, $100 \mathrm{mM}$ Tris (pH 8.5), $1 \mathrm{mM}$ DTT and $50 \mathrm{mM}$ preQ $_{1}$.

\subsection{Structure determination}

X-ray data were collected on an RAXIS-IV image plate system mounted on a Rigaku RU300 rotating anode generator operating at $50 \mathrm{kV}$ and $100 \mathrm{~mA}$, using focusing mirrors (MSC, USA). The crystal to detector distance was $120 \mathrm{~mm}$ and images of $1^{\circ}$ oscillation with a 5 min exposure time were collected at $-173^{\circ} \mathrm{C}$. Diffraction data were processed using the programs DENZO and SCALEPACK [21]. To collect data under cryo-conditions crystals were flash frozen in a solution containing $50 \mathrm{mM}$ HEPES (pH 7.5), 16\% (w/v) PEG 8000 and $20 \%(\mathrm{v} / \mathrm{v})$ glycerol as a cryo-protectant.

The structures were refined by several cycles of energy minimization, using X-PLOR [22] and manual correction using ARP [23] and $\mathrm{O}[24]$.

\section{Results}

\subsection{Construction and purification of TGT(S103A)}

In order to characterize the structure and function of $\mathrm{Ser}^{103}$ in the base exchange reaction, a serine to alanine mutation of $Z$. mobilis TGT was constructed. The mutation S103A was introduced into the $Z$. mobilis tgt overexpression plasmid

Table 1

Oligonucleotides used in mutagenesis

Oligo Sequence $\left(5^{\prime} \text { to } 3^{\prime}\right)^{\mathrm{a}}$

S103A-s CTATTTTGACGGATGCCGGCGGGTATCAG

S103A-a CCTGATACCCGCCGGCATCCGTCAAAAAG

a The mutated codon is underlined.

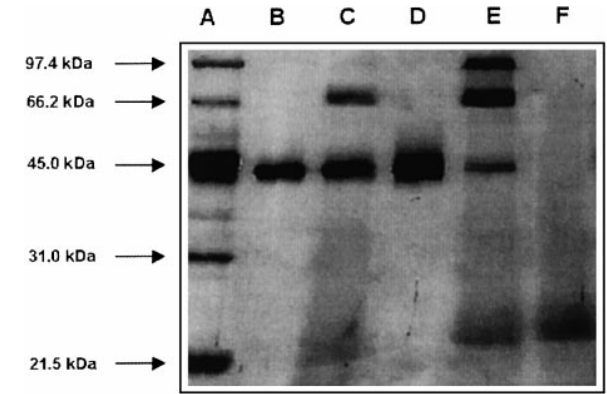

Fig. 1. Silver-stained SDS-PAGE of wild-type TGT and TGT(S103A) in the absence or presence of tRNA ${ }^{\mathrm{Tyr}}-\left(\mathrm{G}_{34}\right)$. Lane A, molecular mass standards; lane B, TGT (wt); lane C, TGT (wt)+tRNA; lane D, TGT(S103A); lane E, TGT(S103A)+tRNA; lane F, tRNA.

pETZM4 [16] by PCR using an optimized method for sitedirected mutagenesis $[17,18]$. To verify successful mutagenesis and exclude potential additional mutations, the complete insert of the mutated plasmid was sequenced. The resulting plasmid pETZM4-S103A was transformed into E. coli BL21(DE3) for overexpression of the mutated tgt gene as described previously [16]. TGT(S103A) could be purified identically to the wild-type TGT, indicating a correct folding of the enzyme.

\subsection{Characterization of the $T G T(S 103 A)$ mutation by activity assay and denaturing gel electrophoresis}

The measurement of TGT(S103A) activity revealed a drastic reduction of activity compared with wild-type Z. mobilis TGT as positive control. Even at high substrate and enzyme concentrations the initial velocities were too low to determine $V_{\max }$ and $K_{\mathrm{M}}$ because of the pronounced errors obtained for the measured values. This finding was consistent with the properties of $E$. coli TGT(S90A) [14]. It has been shown that $E$. coli $\mathrm{TGT}(\mathrm{S} 90 \mathrm{~A})$ binds cognate tRNA under native conditions [14]. To see if $Z$. mobilis TGT(S103A) retained

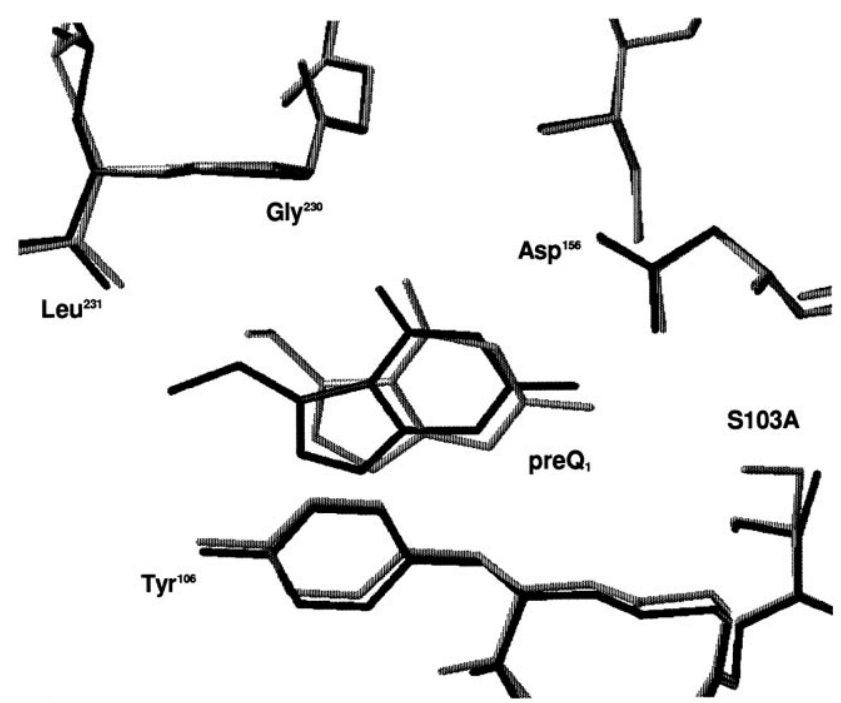

Fig. 2. The alignment of all main chain atoms of wild-type TGT (grey) and TGT(S103A) (black) shows a slight rotation of the preQ ${ }_{1}$ molecule towards the amide of Gly ${ }^{230}$. Additional density indicates a rotation of the aminomethyl group of preQ $_{1}$ by $\sim 95^{\circ}$ in the TGT(S103A) structure, allowing an interaction with the main chain carbonyl oxygen of Leu ${ }^{231}$. 
Table 2

Data collection and refinement statistics

\begin{tabular}{ll}
\hline TGT(S103A) & \\
\hline Space group & $\mathrm{C} 2$ \\
Cell constant $a(\AA)$ & 90.60 \\
Cell constant $b(\AA)$ & 65.04 \\
Cell constant $c(\AA)$ & 70.74 \\
Cell constant $\beta(\mathrm{deg})$ & 96.25 \\
Resolution $(\AA)$ & $71-200$ \\
Wavelength $(\AA)$ & 1.54 \\
Temperature of data collection (K) & 100 \\
No. of reflections & 27018 \\
Completeness of all data (\%) & 97.4 \\
$R_{\text {symm for all data }(\%)}^{\mathrm{a}}$ for & 5.7 \\
Completeness of outer shell (\%) & 95.1 \\
$R_{\text {symm in outer shell }(\%)}$ is & 24.3 \\
$R_{\text {free }}^{\mathrm{b}}(\%)$ & 19.9 \\
$R$-factor $(\%)$ & 16.1
\end{tabular}

${ }^{\mathrm{a}} R_{\text {symm }}=\Sigma|I-\langle I\rangle| / \Sigma I$, where $I$ is the observed intensity and $\langle I\rangle$ is the average intensity for multiple measurements.

${ }^{\mathrm{b}}$ The $R_{\text {free }}$ [26] was calculated from a random selection of reflections constituting $\sim 10 \%$ of the data; the $R$-factor was calculated with the remaining intensities.

the ability to form the covalent protein/tRNA intermediate shown for wild-type TGT, we performed a band-shift experiment under denaturing conditions as described previously [12] using wild-type TGT as positive control. Both wild-type and mutated TGT were incubated at $37^{\circ} \mathrm{C}$ for $1 \mathrm{~h}$ in the presence and absence of a 20 -fold excess of $E$. coli tRNA $^{\mathrm{Tyr}}-(\mathrm{G} 34)$. Analysis by SDS-PAGE clearly showed the presence of shifted bands $(\sim 66 \mathrm{kDa})$ consistent with a TGT monomer $\cdot$ tRNA complex (Fig. 1). Surprisingly, an additional band at a higher

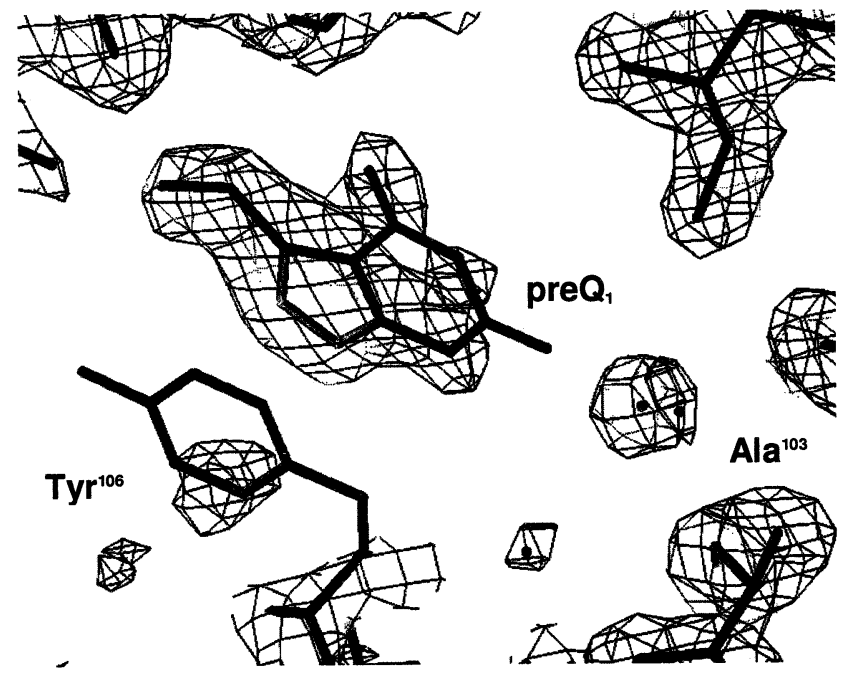

Fig. 3. Representation of the $2.0 \AA$ resolution $2 f_{\mathrm{o}}-f_{\mathrm{c}}$ map contoured at $1.0 \sigma$ of the TGT(S103A) binding pocket in complex with $\mathrm{preQ}_{1}$. Electron density of the refined structure is partially missing for the $\mathrm{C} 2-\mathrm{NH}_{2}$ group of the $\mathrm{preQ}_{1}$ molecule and for the side chain of $\mathrm{Tyr}^{106}$, suggesting a reduced occupancy of the substrate in the binding pocket.

molecular weight $(\sim 97 \mathrm{kDa})$ was observed in the case of TGT(S103A) seen also for Z. mobilis TGT(D156A) [12], for which no explanation can be given. Nevertheless, the results confirm that TGT(S103A) is able to form a covalent intermediate with the tRNA and that Ser ${ }^{103}$ cannot be the active site nucleophile involved in the formation of a covalent bond to tRNA. Consistent with these results, we also find that the

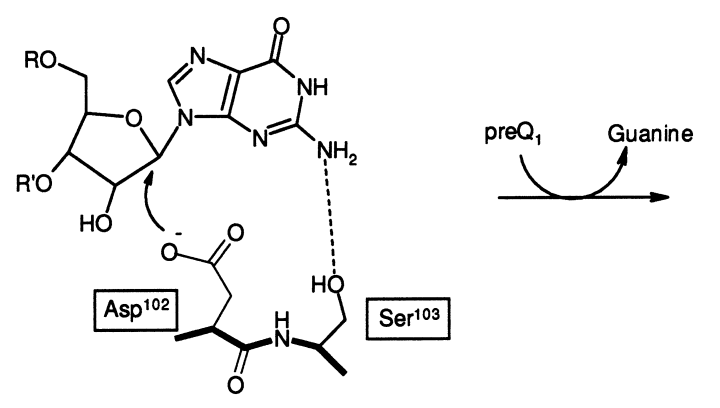

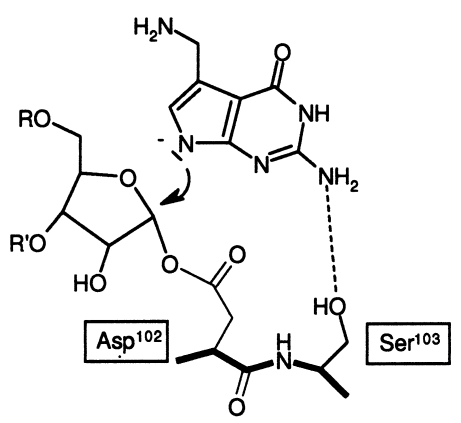

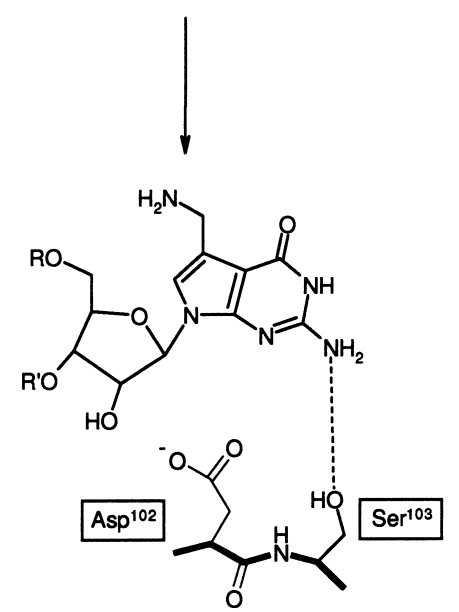

Fig. 4. Proposed catalytic mechanism with $\mathrm{Asp}^{102}$ as the active site nucleophile. The nucleophilic attack of $\mathrm{Asp}^{102}$ at $\mathrm{Cl}^{\prime}$ atom of the guanosine results in a covalent TGT/tRNA intermediate. Subsequent nucleophilic substitution by the deprotonated preQ $\mathrm{Q}_{1}$ molecule preserves the $\beta$-configuration and leads to the modified preQ $\mathrm{Q}_{1}$-tRNA. The residue $\mathrm{Ser}^{103}$ performs a critical role in substrate orientation. 
E. coli TGT(S90A) mutant forms a covalent complex with both full-length tRNA and minihelix RNA (data not shown).

\subsection{Structural characterization}

Soaking crystals of TGT(S103A) with preQ $_{1}$ and subsequent structure analysis was used to determine the binding mode of the substrate. The X-ray structure has been solved at $2.0 \AA$ resolution (Table 2) and shows only minor changes compared to the wild-type structure (Fig. 2). The serine to alanine mutation is clearly confirmed by the electron density map. A preQ $Q_{1}$ molecule could easily be fit in the difference electron density. The $2 f_{\mathrm{o}}-f_{\mathrm{c}}$ electron density map of the refined TGT(S103A) structure including the substrate clearly indicates a similar orientation of the preQ $_{1}$ molecule as observed in the wild-type structure [11]. The base is intercalated between the side chains of $\mathrm{Met}^{260}$ on one side, and $\mathrm{Tyr}^{106}$ and $\mathrm{Cys}^{158}$ on the other side. Specific recognition is allowed through hydrogen bonding between the side chain of Asp ${ }^{156}$ and the $1-\mathrm{NH}$ and $2-\mathrm{NH}_{2}$ groups, and between the amide of $\mathrm{Gly}^{230}$ and oxygen $\mathrm{O} 6$. These residues are strictly conserved in all presently known TGT sequences of prokaryotes, archaebacteria and eukaryotes, suggesting a common guanine recognition motif [15]. Interestingly, difference electron density indicates a rotation of the exocyclic methylene-amino group of preQ $_{1}$ by $\sim 95^{\circ}$ in the TGT(S103A) complex, allowing an interaction with the main chain carbonyl oxygen of $\mathrm{Leu}^{231}$ (Fig. 2). The 2- $\mathrm{NH}_{2}$ group of $\mathrm{preQ}_{1}$ forms a hydrogen bond $(2.8 \AA)$ to an additional water molecule in the vicinity of the serine to alanine mutation. This water molecule is not present in the wild-type structure, and thus formally replaces the $\mathrm{OH}$ functional group of $\mathrm{Ser}^{103}$. In the wild-type TGT/preQ ${ }_{1}$ complex, the $2-\mathrm{NH}_{2}$ group is involved in a weak hydrogen bond $(3.5 \AA)$ to $\mathrm{Ser}^{103}$, suggesting that this residue assists in the correct orientation of the substrate required for the base exchange reaction [11]. In the present complex, no electron density was observed for the $\mathrm{C} 2-\mathrm{NH}_{2}$ group of $\mathrm{preQ}_{1}$ in the $2 f_{\mathrm{o}}-f_{\mathrm{c}}$ map at a contour level of $1.0 \sigma$ (Fig. 3), nor could electron density be properly assigned to the hydroxyphenyl ring of $\mathrm{Tyr}^{106}$, indicating that this residue is not fully occupied in the position adjacent to the preQ $_{1}$ molecule (Fig. 3). A superposition based on all main chain atoms between wildtype TGT and TGT(S103A) shows a slight rotation of the preQ $_{1}$ molecule towards the amide group of $\mathrm{Gly}^{230}$ (Fig. 2).

\section{Discussion}

The results presented here support the catalytic mechanism proposed on the basis of structural and mutagenesis studies described previously $[11,12]$. The X-ray structure of queuosine monophosphate shows that the $\beta$-configuration of the ribose is preserved [25]. Consequently, two consecutive $S_{N} 2$ reactions are most likely involved in the base exchange reaction catalyzed by TGT as indicated earlier $[11,12]$. In this mechanism, a covalent TGT/tRNA intermediate is formed as a consequence of the preceding nucleophilic attack by the carboxylate of $\mathrm{Asp}^{102}$ at the $\mathrm{Cl}^{\prime}$ atom of the wobble guanosine (Fig. 4). After replacement of guanine by $\mathrm{preQ}_{1}$ at the binding site, the deprotonated $\mathrm{preQ}_{1}$ attacks carbon $\mathrm{Cl}^{\prime}$ of the ribose, resulting in the final preQ $_{1}$-modified tRNA. In our band-shift experiment, we have demonstrated that TGT(S103A) still binds covalently to tRNA, in agreement with the above postulated mechanism. Therefore, residue $\operatorname{Ser}^{103}$ cannot be involved in the nucleophilic attack of the wobble guanosine. In the enzyme assay, we observed a dramatic reduction of activity of Z. mobilis TGT(S103A) compared to the wild-type TGT. However, total inactivation upon mutation of the $Z$. mobilis TGT Ser ${ }^{103}$ to alanine could not be determined, consistent with the results obtained for E. coli TGT(S90A) [14]. The $\mathrm{X}$-ray structure of $Z$. mobilis TGT(S103A) showed difference density for a preQ $Q_{1}$ molecule, suggesting a similar binding mode to that observed for wild-type TGT. This clearly implies that the wobble guanine is still recognized in the $\mathrm{preQ}_{1}$ binding pocket of TGT(S103A). However, the lack of well resolved electron density attributable to the $\mathrm{C} 2-\mathrm{NH}_{2}$ group of $\mathrm{preQ}_{1}$ and to the side chain of $\mathrm{Tyr}^{106}$ in the refined structure of TGT(S103A) indicates an elevated flexibility of the preQ $\mathrm{Q}_{1}$ molecule in the binding pocket. In the wild-type TGT, the 2- $\mathrm{NH}_{2}$ group of $\mathrm{preQ}_{1}$ forms a weak hydrogen bond (3.5 $\AA$ ) to $\operatorname{Ser}^{103}$ [11]. This interaction could be crucial for immobilizing the wobble guanosine in a binding position suitable for the nucleophilic attack and tighter binding of the intermediate transition state with $\operatorname{Asp}^{102}$ (Fig. 4). Removal of the Ser ${ }^{103}$ hydroxyl group by an alanine replacement is accompanied by a change to a more hydrophobic environment. In the refined TGT(S103A) structure, an additional water molecule is detected in the vicinity of $\mathrm{Ala}^{103}$, allowing for an extra hydrogen bond $(2.8 \AA)$ to the $2-\mathrm{NH}_{2}$ group of $\mathrm{preQ}_{1}$. This water molecule could mimic the Ser-OH recognition site, however it obviously does not retain the orientational fixation of the $\mathrm{Ser}^{103}$ hydroxyl group binding to the $2-\mathrm{NH}_{2}$ group of the wobble guanosine. We therefore conclude that $\operatorname{Ser}^{103}$ plays a critical role in the appropriate immobilization and orientation of the substrate for the base exchange reaction.

Acknowledgements: This work was supported by the Deutsche Forschungsgemeinschaft (Grant: KL 1204/1-1 and SFB 286).

\section{References}

[1] Okada, N. and Nishimura, S. (1979) J. Biol. Chem. 254, 30613066.

[2] Slany, R.K. and Kersten, H. (1994) Biochimie 76, 1178-1182.

[3] Goodman, H.M., Abelson, J., Landy, A., Brenner, S. and Smith, J.D. (1968) Nature 217, 1019-1024.

[4] Harada, F. and Nishimura, S. (1983) Biochemistry 11, 302308.

[5] Katze, J.R., Basile, B. and McCloskey, J.A. (1982) Science 216, $55-56$.

[6] Katze, J.R., Gündzün, U., Smith, D.L., Chen, C.S. and McCloskey, J.A. (1984) Biochemistry 23, 1171-1176.

[7] Persson, B.C. (1994) Mol. Microbiol. 8, 1011-1016.

[8] White, B.N., Tener, G.M., Holden, J. and Suzuki, T. (1973) J. Mol. Biol. 74, 635-651.

[9] Dirheimer, G., Baranowski, W. and Keith, G. (1995) Biochimie 77, 99-103.

[10] Durand, J.M., Okada, N., Tobe, T., Watarai, M., Fukuda, I., Suzuki, T., Nakata, N., Komatsu, K., Yoshikawa, M. and Sasakawa, C. (1994) J. Bacteriol. 176, 4627-4634.

[11] Romier, C., Reuter, K., Suck, D. and Ficner, R. (1996) EMBO J. $15,2850-2857$.

[12] Romier, C., Reuter, K., Suck, D. and Ficner, R. (1996) Biochemistry $35,15734-15739$.

[13] Nogushi, S., Nishimura, Y., Hirota, Y. and Nishimura, S. (1982) J. Biol. Chem. 257, 6544-6550

[14] Reuter, K., Chong, S., Ullrich, F., Kersten, H. and Garcia, G.A. (1994) Biochemistry 33, 7041-7046.

[15] Romier, C., Meyer, J.E.W. and Suck, D. (1997) FEBS Lett. 416, 93-98.

[16] Reuter, K. and Ficner, R. (1995) J. Bacteriol. 177, 5284-5288. 
[17] Ho, S.N., Hunt, H.D., Horton, R.M., Pullen, J.K. and Pease, L.R. (1989) Gene 77, 51-59.

[18] Ansaldi, M., Lepelletier, M. and Mejean, V. (1996) Anal. Biochem. 234, 110-111.

[19] Akimoto, H., Imamiya, E., Hitaka, T., Nomura, H. and Nishimura, S. (1988) J. Chem. Soc. Perkin Trans. 1, 1638-1644.

[20] Romier, C., Ficner, R., Reuter, K. and Suck, D. (1996) Proteins Struct. Funct. Genet. 24, 516-519.

[21] Otwinowski, Z. (1993) DENZO, Yale University, New Haven, CT.
[22] Brünger, A.T. (1992) XPLOR, Version 3.4, Yale University, New Haven, CT.

[23] Jones, T.A., Zou, J.Y., Cowan, S.W. and Kjeldgaard, M. (1991) O Acta Cryst. A47, 110-119.

[24] Lamzin, V.S. and Wilson, K.S. (1993) ARP Acta Cryst. D49, $129-147$.

[25] Yokoyama, S., Miyazawa, T., Iitaka, Y., Yamaizumi, Z., Kasai, H. and Nishimura, S. (1979) Nature 282, 107-109.

[26] Brünger, A.T. (1992) Nature 355, 472-474. 